\title{
Lectura y conocimiento en Bibliotecología
}

\author{
Héctor Guillermo Alfaro López ${ }^{\star}$
}

Artículo recibido:

3 de diciembre de 2020

Artículo aceptado:

23 de agosto de 2021

Artículo de investigación

\section{RESUMEN}

Se busca comprender cómo se despliega cognoscitivamente la lectura a través del campo bibliotecológico, principalmente en la educación y la investigación, para lo cual se implementan los conceptos mediadores, intermediadores y traducción del sociólogo Bruno Latour. Los intermediadores consisten en las acciones que transportan contenidos sin que sufran cambios; así, la lectura concebida como intermediador ha predominado en el campo bibliotecológico y además está determinada por la objetividad. Los mediadores transforman la significación de sus contenidos al pasar por la traducción. La lectura como mediador conlleva la transformación de la información en conocimiento

* Instituto de Investigaciones Bibliotecológicas y de la Información, Universidad Nacional Autónoma de México, México galfaro@unam.mx

INVESTIGACIÓN BIBLIOTECOLÓGICA, vol. 35, núm. 89, octubre/diciembre, 2021, México, ISSN: 2448-8321 pp. 79-90 
diferente o nuevo al ser traducido por mediación del planteamiento de problemas y preguntas al texto, lo cual propicia una lectura signada por la subjetividad y que puede constituirse en factor de transformación cognoscitiva del campo bibliotecológico.

Palabras clave: Lectura; Conocimiento; Mediador; Intermediador

\title{
Reading and knowledge in Library Science Héctor Guillermo Alfaro López
}

\begin{abstract}
The intention here is understanding how reading develops cognitively through the Library Science field, mainly in its education and research dimensions, resorting to the following concepts of sociologist Bruno Latour: mediators, intermediaries and translation. The intermediaries consist of the actions that transport content without undergoing changes, so that the reading (conceived as an intermediary) has predominated in the Library Science field and besides is also determined by objectivity. The mediators transform the meaning of its contents by undergoing translation. Reading as a mediator entails the transformation of information into different or new knowledge as it is translated, through the posing of problems and questions to the text, all of which encourages a reading marked by subjectivity, which can become a transforming factor in the Library Science field.
\end{abstract}

Keywords: Reading; Knowledge; Mediator; Intermediary

\section{INTRODUCCIÓN}

T temática de la lectura sólo hasta un momento relativamente reciente ha ocupado un lugar preponderante y consolidado entre los temas tradicionales y canonizados de la Bibliotecología. Eso ha permitido avizorar la extrema complejidad de esta práctica, que de ninguna manera se reduce a una mera descodificación de la textualidad, con lo cual pone de manifiesto 
su posición estratégica dentro del conocimiento bibliotecológico: toda la actividad bibliotecológica y bibliotecaria tiene un objetivo, lo que le da su sentido, satisfacer las necesidades de información de la sociedad; pero de manera individual y concreta las personas acceden a esa información que se les brinda por mediación de la lectura.

Tal posicionamiento de la temática de la lectura ha estimulado una vasta y variada producción investigativa con lo que se ha ampliado el conocimiento de esta práctica dentro de la disciplina bibliotecológica; sin embargo, en la vastedad de esta producción de indagación de diversa índole sobre la lectura, queda de manifiesto una desproporción entre la gran mayoría de investigaciones que se deslizan sobre la superficie del tema y aquellas, escasas, que buscan dar un soporte más sólido, fundando conceptual y teóricamente la temática de la práctica de la lectura dentro del campo bibliotecológico.

Es previsible que en la medida en que más investigaciones se elaboren desde esta última perspectiva, contribuirán a su vez a dar fundamento bibliotecológico a este tema crucial, con lo que por añadidura se contribuye a la fundamentación científica del campo bibliotecológico. De ahí la pertinencia, en este caso, de abordar la relación de lectura y conocimiento en Bibliotecología a partir de una serie de conceptos que explican cómo se ha desplegado la lectura en los procesos cognoscitivos del campo bibliotecológico a lo largo de su desenvolvimiento histórico.

\section{MÉTodo}

Al iniciar el recorrido de esta indagación es preciso dejar establecido que se plantea desde un enfoque claramente epistemológico. Desde este enfoque se enuncia el problema que orienta la reflexión: ¿cuál es el rol de la práctica de la lectura en los procesos cognoscitivos en el campo bibliotecológico? Para dar respuesta a esta cuestión, por método, se establece primeramente el contexto: el campo bibliotecológico que, en cuanto campo de conocimiento, se encuentra integrado por un "conjunto de regiones" que conceptualmente pueden definirse como prácticas globales, como son investigación, biblioteca, educación bibliotecológica, asociaciones de bibliotecarios y publicaciones, entre otras.

A través de todas las prácticas globales circula y se manifiesta la práctica de la lectura de manera específica, de acuerdo a las características de cada una de las regiones mencionadas. Mas, es en la práctica global de la educación donde la lectura asume una presencia iniciática, que será el soporte de su desenvolvimiento a través de las otras regiones del campo. La base que 
sustenta el proceso educativo bibliotecológico son los saberes, que encuentran cauce y plasmación en los diversos soportes y formatos de los textos, pero que aquí serán conjuntados y subsumidos en la categoría genérica de libro. A continuación se explica cómo se correlaciona la lectura en la educación con la lectura en la investigación bibliotecológica.

\section{Conceptos}

En el orden metódico, conforme se da explicación del desenvolvimiento de la práctica de la lectura en el campo bibliotecológico, se van exponiendo e instrumentalizando los conceptos centrales que dan explicación de las distinciones que asume la lectura a lo largo de ese desenvolvimiento.

Los conceptos que se implementan han sido tomados de la TAR, teoría del actor red, desarrollada por el sociólogo francés Bruno Latour, así como de la hermenéutica de Hans-Georg Gadamer y de la pedagogía de Paulo Freire; aunque estas dos últimas no se encuentren abiertamente ante los reflectores son cruciales para la comprensión del uso de los conceptos elegidos de la TAR. Es de subrayar que, para evitar una interpretación sesgada o limitada respecto al uso que aquí se hace de los conceptos de las mencionadas teorías, el proceder que se ha seguido es crítico-creativo. Lo que significa que se no trata, por ejemplo, de hacer un estudio exhaustivo de la TAR ni de los conceptos en ella desarrollados (que contiene más conceptos de los que aquí se implementan), lo que estaría totalmente fuera de la concepción y objetivo de este artículo. Un proceder crítico-creativo se refiere a la resignificación creativa de los conceptos. Por poner un ejemplo: el sociólogo francés Pierre Bourdieu tomó de Marx el concepto de capital, de sentido económico, y de manera crítico-creativa le dio un nuevo contenido epistemológico, resignificándolo al ampliar el rango explicativo del concepto, de manera que para él el capital pasaba a ser también capital simbólico o político o social. Los conceptos no son entidades fijas ni canonizadas ya que pueden correr el riesgo de esclerotizarse. Siguiendo ese procedimiento, aquí se hacen interactuar los conceptos tomados de la TAR con los elementos aportados por las teorías de Gadamer y Freire. Y es con esa conjunción de elementos conceptuales que debe ser comprendido el desarrollo del tema aquí tratado, el cual, en cuanto tal, es un escorzo de una teoría más amplia que desde años atrás se ha venido elaborando, la teoría de la constitución y autonomía del campo bibliotecológico (Alfaro López, 2010; 2018; 2016), cuyos conceptos medulares son el basamento sobre el que se despliega la argumentación de este artículo. 


\section{Presentación}

Michel Foucault explicó que los saberes son cúmulos de conocimientos ya que son creación de una práctica discursiva y, en cuanto tales, son condición de posibilidad de la ciencia. Pero el saber no se reduce a la ciencia (Foucault, 2009: 9). De manera más explícita: el saber es sobre lo que se habla en la práctica discursiva que, para nuestro caso, es todo aquello que se refiere a la bibliotecología; son los objetos propios del campo y que se les ha dado estatuto científico; es lo referido al campo y los discursos que sobre los objetos se expresan en él, esto es, en las diversas regiones que lo componen; son también la conjunción de enunciados y proposiciones, conceptos y teorías que se aplican en el conocimiento bibliotecológico y, por último, es la utilización de las posibilidades que ofrece el discurso, en nuestro caso, bibliotecológico.

Así es como a lo largo de su proceso formativo los futuros bibliotecólogos reciben el saber propio de su campo que ha quedado plasmado en los libros. En tales libros el saber que circula en el campo bibliotecológico encuentra concreción y con ello la configuración de ciencia, en versión estándar y canónica. En su famoso libro La estructura de las revoluciones científicas, $\mathrm{Ku}-$ hn (2013) da razón de cómo ese tipo de textos son la instancia a través de la que se forman las sucesivas generaciones de científicos, aprendiendo los rudimentos de las ciencias, pero con ello dan también una imagen de la ciencia empobrecida, poco adecuada para su complejidad. Esta clase de libros esquematiza y simplifica el saber, dando una versión estabilizada y uniforme de la ciencia. Es por mediación de la lectura que el saber, constituido en ciencia, puede convertirse en conocimiento; lo cual significa que más allá de los procesos cognoscitivos que acompañan a la lectura, en su producción de conocimiento en sí misma, conlleva elementos que permiten esa generación de conocimiento; y no es, por consiguiente, sólo una técnica neutra que únicamente vehiculiza el saber de la ciencia.

La lectura llevada a cabo en educación bibliotecológica significa el primer acceso orgánico y sistemático al conocimiento bibliotecológico que signará las pautas de lectura normalizada de los sujetos posicionados en las diversas regiones del campo bibliotecológico en su fase de constitución. Esto conduce preguntarse ¿cómo es que esta clase de lectura circula entre los sujetos y las distintas regiones?, ¿cuáles son sus implicaciones en el campo bibliotecológico? El hecho de plantear tales cuestiones nos pone en el lugar donde se bifurcan los caminos entre los hechos del devenir histórico (pasado-presente) y los posibles del porvenir del campo bibliotecológico y, con ello, los desplazamientos pasado-presente-futuro de la práctica de lectura. Para dar respuesta a las preguntas planteadas se seguirá primero la vía de los hechos que vienen 
del pasado y desembocan actualizándose en el presente, para después seguir el camino de los posibles en el porvenir.

Para seguir el desplazamiento de la lectura a través del campo bibliotecológico se implementan algunos de los conceptos de la sociología de Bruno Latour debido a su carácter relacional y fluido: intermediario y mediador-traducción. Este sociólogo francés define sus conceptos a partir de su concreción dinámica explicando que un intermediario es una entidad que transporta significado, pero no genera transformación, lo cual resulta predecible respecto a los datos que vehiculiza, por lo que no importa lo complicado que pueda ser y por ello puede representar algo o nada, eso lo hace olvidable. Por la parte del mediador éste es heterogéneo en su cualidad de transportar múltiples significados, sus datos de entrada y salida son impredecibles; transfigura y cambia el significado de los elementos con que se expresa su capacidad para llevar a cabo la traducción (concepto también central de Bruno Latour, del que se dará explicación y hará uso más adelante), esto es, la transformación de aquello que es transportado. En palabras del propio Latour:

Un intermediario, en mi vocabulario, es lo que transporta significado o fuerza sin transformación: definir sus datos de entrada basta para definir sus datos de salida. Para todo propósito práctico un intermediario puede considerarse no sólo una caja negra sino también una caja negra que funciona como una unidad, aunque internamente esté compuesta de muchas partes. Los mediadores, en cambio, no pueden considerarse sólo uno; pueden funcionar como uno, nada, varios o infinito. Sus datos de entrada nunca predicen bien los de salida; su especificidad debe tomarse en cuenta cada vez. Los mediadores transforman, traducen, distorsionan y modifican el significado o los elementos que se supone que deben transportar. No importa lo complicado que sea un intermediario, puede representar, para todo propósito práctico, una unidad o incluso nada porque puede ser fácilmente olvidado. Por simple que pueda parecer un mediador, puede volverse complejo; puede llevar en múltiples direcciones que modificarán todas las descripciones contradictorias atribuidas a su rol. (Latour, 2008: 63)

Queda así de manifiesto su complejidad multidireccional. Los términos de intermediario y mediador, en cuanto conceptos de tipo relacional que expresan movimiento vehiculizador de contenidos, relacionan tanto a sujetos, acciones y objetos que según el flujo de su movimiento por momentos se presentan en un primer plano haciéndose abiertamente visibles, para un instante después retroceder a un segundo plano, interrumpiendo la transmisión de datos de que son portadores (Latour, 2008: 48-67). Los susodichos conceptos marcan el punto donde los caminos se separan, como se había enunciado líneas atrás, entre los intermediarios que nos dan explicación del desplazamiento de la lectura en el 
pasado y el presente, y los mediadores que nos proyectan a las posibilidades de la lectura futura en el campo bibliotecológico.

Veamos en primera instancia la trayectoria que sigue el intermediario: la práctica de la lectura es un intermediario que se relaciona con el objeto libro, que en sí mismo es un intermediario. La lectura en cuanto intermediario transporta significado pero sin transformación; el carácter de los propios datos con que se le da inicio definen los datos posteriores de salida de ella. Esto es debido a que el libro, con el que se lleva una relación de intermediario, contiene el saber normalizado que sustenta a la ciencia bibliotecológica asimismo normalizada (en terminología de Thomas Kuhn), la cual tiene un marcado sustrato técnico que también es un factor que signa con su impronta a la lectura. Al leer el libro se desenvuelven como intermediarios lectura-libro, pero no se da entre ambos la traducción (como explica Bruno Latour), no produce modificación de significado o de los elementos que se supone deben transportar ambos para producir conocimientos nuevos o diferentes.

En otras palabras, los saberes con los que de antemano cuenta el lector para llevar a cabo la lectura no sufren cambio, traducción, para dar lugar a un conocimiento bibliotecológico diferente o nuevo. Sólo se reiteran, transportan, los conocimientos previamente establecidos y estabilizados (técnicamente), propios de un capital de conocimiento constituido y estatuido en el campo bibliotecológico. La lectura como intermediario se despliega a través del campo; habiendo adquirido ese estatuto originariamente en la educación, se manifiesta de esa manera en el resto de las regiones del campo. En la región de investigación la lectura y los libros adquieren su pleno estatuto cognoscitivo, puesto que transportan significado, conocimiento, sin transformación; pero con tal estatuto (intermediario) se contribuye a legitimar epistemológicamente el conocimiento ya establecido y consolidado, con su basamento técnico. Con ello pone en evidencia que es un conocimiento objetivado y estático, que se corresponde ceñidamente con una lectura en cuanto intermediario, llevada a cabo por un sujeto normalizado y objetivado.

La lectura de un texto de ciencia enfrenta a una información que se pretende es el resultado de una investigación precisa y rigurosa en observación y experimentación. Y, además, se encuentra escrito en una prosa austera, encorsetada, sin puntos de fuga hacia la subjetividad, donde los conceptos obturan el paso a las metáforas y las imágenes: escritura donde señorea la primera persona del plural. El lector, por su parte, tiene que mediar su lectura con los códigos que le brinda un texto de semejante índole para hacérselo legible. Ello implica que ha de contar con elementos previos del saber de la ciencia y aceptar las señalizaciones de objetividad a lo largo de la ruta que marca el texto. Pero la lectura del texto científico no se reduce a la esfera de 
comprensión del contenido, sino que también retroactúa en otro nivel sobre el lector: lo transfigura, constituyéndolo como un lector objetivado, normalizado de acuerdo al orden racionalista establecido por el texto, que a su vez es resultado de una ciencia normalizada bajo el designio de objetividad y que se encuentra en condición de llevar a cabo la lectura de libros de su ciencia como intermediario dentro del campo de conocimiento.

Una vez llegados a este límite es pertinente retornar al punto en que los caminos se separaban, para ahora seguir la otra senda, de los mediadores; $c a-$ minemos hacia el horizonte de los posibles. Recordemos cómo Bruno Latour caracteriza a los mediadores: "[...] sus datos de entrada nunca predicen bien los de salida; su especificidad debe de tomarse en cuenta cada vez. Los mediadores transforman, traducen, distorsionan y modifican el significado con los elementos que se suponen deben transportar" (Latour, 2008: 63). La práctica de la lectura comprendida como mediador es aquella que al ser un proceso de relación con un libro-texto brinda una información: hay datos de entrada pero no pueden predecirse bien los datos o más exactamente los conocimientos que se producen de salida una vez que ya se ha realizado el acto de lectura, ello en razón de que se da la traducción entre la lectura y el libro transformando el significado que se supone transporta el texto, a diferencia de la lectura en cuanto intermediario que implica continuidad, pero ésta aparentemente sencilla interpretación de la lectura como mediador conlleva una gran complejidad ya que en ella, además, se ventilan arduos procesos cognitivos, que a su vez entrañan procesos de transfiguración del propio campo bibliotecológico en su conjunto, por lo que se requiere dar precisa explicación de ello.

El concepto clave en el despliegue de los mediadores es el de traducción, ya que expresa el momento disruptivo que altera o cambia el movimiento, la acción, dándoles una orientación cualitativa distinta a los contenidos, en este caso de la lectura y el libro. Qué viene a significar la traducción dentro de la lectura en cuanto mediador: el planteamiento de problemas y su enunciación en preguntas. Todo lo contrario a una lectura que reitera, sin cuestionar, una información establecida y canonizada, y que en cuanto tal, funge como reafirmación de semejante canonización. La práctica de la lectura, mediador, al desplegarse sobre el libro plantea y/o encuentra problemas en el texto por conducto de las preguntas. Pero para que la lectura transite por el espíritu de la pregunta, la cual es vehiculizada en la traducción, hay condicionantes que respaldan a la lectura: los saberes.

A diferencia del saber que viene a ser el significado (elementos) que vehiculiza la lectura como intermediario, cuyo carácter es especializado y reiterativo de un conocimiento establecido, la lectura en cuanto mediador ha de estar integrada por saberes múltiples y cambiantes. El bibliotecólogo ha 
de estar respaldado en la lectura de sus textos bibliotecológicos por libros de distintas disciplinas: filosofía, literatura, historia, sociología, etc. Bagaje cognitivo cultural que al fusionarse con el saber bibliotecológico permite su ampliación de visión respecto a la realidad social sobre la que actúa. Pero, más aún, el aporte de tales disciplinas propicia la manifestación de la imaginación y la creatividad que pueden galvanizar la práctica de la lectura. De hecho ese impulso imaginativo y creativo es el que se llega a desenvolver en aquella lectura que plantea problemas y preguntas críticas innovadoras, revulsivas al texto.

De esta manera se puede establecer, como plantea Hans-Georg Gadamer, un diálogo con el texto, un diálogo en el que el constante plantear preguntas es el pasaporte infaltable para recorrer todos los territorios del libro (Gadamer, 1993). Las preguntas sacan de ese mutismo en que la lectura como intermediario deja al texto, para hacer que éste asimismo nos interpele y con ello que cuestionemos la certeza de nuestros saberes. Por otra parte, es de indicar que una lectura de tal índole implica que las preguntas estén marcadas por el riesgo; como explican Paulo Freire y Antonio Faundez: al estimular la invención rompen con las formas establecidas, burocráticas y seguras de conocimiento (Freire y Faundez 2013), propiciando la generación de conocimientos nuevos, diferentes, alternativos.

Ahora bien, conforme la lectura-mediador se despliega, una presencia la acompaña en las sucesivas traducciones por las que cruza: la subjetividad. Esa presencia que fue exiliada, incluso, anatemizada desde los orígenes de la ciencia occidental moderna, y que como desvaída sombra fue arrinconada en los suburbios de expresiones de saber que se estimaba no ofrecían la verdad, como las Humanidades, las Artes y las Ciencias Sociales. La objetividad se erigió en el inquisidor que condenó al exilio a la subjetividad. Pero el proyecto histórico de conformación de la objetividad comenzó a desgastarse en las décadas finales del siglo pasado, lo que implicó la exhumación y el retorno de la subjetividad al escenario principal del conocimiento, mas ya no con el antiguo atuendo, sino con un rostro renovado: no es una entidad estática ni exclusivamente recluida en el espacio de los sentidos sino, por el contrario, como la caracteriza Michel Foucault, es moviente mutable y puede producir un conocimiento también verdadero.

Es claro que la práctica lectora, mediador, es llevada a cabo por un lector constituido en cuanto subjetividad, pero a su vez esta clase de lectura es instituyente de subjetividad: es un movimiento recursivo donde simultáneamente ambos son causante y causado. La fuerza motriz de este movimiento recursivo es la imaginación y la creatividad producto de la fusión e interacción de saberes diversos, lo que termina por resquebrajar la figura monolítica y pétrea del lector normalizado y objetivado, que pone en práctica la lectura 
como intermediario y que resulta ser racionalista y descorporizado, reproductor y legitimador de un capital de conocimiento, establecido y fundado técnicamente. Por otra parte, para que la lectura-mediador muestre todo su potencial transformador, ha de comprenderse a través de su desenvolvimiento en la región de investigación del campo bibliotecológico.

Cuando se hacía el seguimiento de la lectura como intermediación, partiendo de la región de educación bibliotecológica, se explicaba cómo esa trayectoria de lectura en la investigación se convierte en legitimadora epistemológica de un conocimiento establecido y canónico, en el que el cuestionamiento no tiene una posición reconocida. Por el contrario, la práctica de lectura, mediador, con sus consiguientes traducciones a través de problemas y preguntas, rompe a su paso las barreras que impone un conocimiento estático, reiterativo y técnico para desembocar en el océano de un conocimiento diferente e innovador. El proceso de investigación, que tiene su antesala en la lectura de los libros que contienen la ciencia bibliotecológica, al estar marcado por la lectura como mediador conlleva la problematización perenne, la puesta en marcha de preguntas. Esto da lugar a una reflexividad imaginativa y creativa que, inclusive, puede hacer uso extensivo e intensivo de la construcción conceptual y teórica de objetos y prácticas para fundamentarlos bibliotecológicamente (Alfaro López, 2018). Al tener esta ruta de lectura el respaldo de los saberes de otras disciplinas ofrece elementos cognoscitivos de apoyo para llevar a cabo crítica y creativamente dicha construcción epistemológica.

Como mera acotación, la lectura, mediador, confluye a su vez en otro mediador: la escritura, lo que conlleva que igualmente se despliegue a través de varias instancias de traducción. La escritura como mediador implica la concreción de investigación crítica conducida por la subjetividad cognoscente. Escribir bajo la directriz investigativa ha de conllevar mediaciones, supuestos y desenvolvimientos reflexivos y críticos, que son galvanizados por el planteamiento de problemas y preguntas: la escritura de semejante índole no ha de ser una mera redacción y reiteración de saberes y conocimientos ya establecidos. Lectura y escritura en cuanto mediadores son los posibles que pueden ser un agente de impulso para que el campo bibliotecológico transite de su fase de constitución hacia su fase de autonomía, de plena cientificidad.

\section{Conclusión}

La práctica de lectura tiene una notable preponderancia dentro de un campo de conocimiento debido a su posición distintiva y estratégica: es la instancia a través de la cual se tiene acceso a los saberes (información) específicos, en 
nuestro caso, propios y definitorios del campo bibliotecológico; asimismo, la lectura en cuanto mediador contribuye para llevar a cabo la transformación de los saberes en conocimiento y sobre todo de manera dirigida cuando está surcada por problemas y su enunciación en preguntas, puesto que son factores que estimulan la creatividad. Creatividad que se convierte en impulso hacia la generación de conocimiento diferente o innovador cuando la lectura de los saberes bibliotecológicos se encuentra, además, respaldada por saberes provenientes de diversas disciplinas. Esto pone de manifiesto la especificidad y complejidad de los procesos cognoscitivos que se realizan en las distintas regiones del campo, en especial en la práctica global de investigación. $\mathrm{Pa}$ ra hacer legibles tales procesos, que se realizan por medio de la lectura, se requiere un abordaje de índole conceptual y teórico. Los conceptos ofrecen una visión abstracta y concatenada de procesos empíricos, lo que permite una comprensión unificada de ellos.

La teoría, al ser un sistema estructurado de conceptos, se configura como una concepción y conocimiento multidimensional de la realidad, de la cual no debe estar exenta la imaginación creativa. Con ello se abre también la vía para emprender la fundamentación de la práctica de la lectura como objeto de conocimiento bibliotecológico, y cómo tal práctica, a su vez, puede convertirse en un factor, incluso, decisivo para la transformación del campo bibliotecológico.

Agradecimientos Agradezco al Programa de Apoyos para la Superación del Personal Académico de la UNAM (PASPA), por el apoyo otorgado durante la estancia de investigación en la Escuela Interamericana de Bibliotecología de la Universidad de Antioquia, Medellín Colombia, donde se llevó a cabo el presente trabajo.

\section{REFERENCIAS}

Alfaro López, Héctor Guillermo. 2010. Estudios epistemológicos de bibliotecología. México: UNAM, Centro Universitario de Investigaciones Bibliotecológicas.

Alfaro López, Héctor Guillermo. 2016. "Problemas en la construcción de la imagen y la lectura de imagen como objetos de conocimiento en el campo bibliotecológico", en Hacia la construcción de la imagen y su lectura como objetos de conocimiento en Bibliotecología, coordinado por Héctor Guillermo Alfaro López y Graciela Leticia Raya Alonso, 1-30. México: UNAM-IIBI.

Alfaro López, Héctor Guillermo. 2018. Construcción epistemológica de la imagen y la lectura de imagen como objetos de conocimiento en el campo bibliotecológico. México: UNAM, Instituto de Investigaciones Bibliotecológicas y de la Información. 
Foucault, Michel. 2009. La arqueología del saber. México: Siglo XXI.

Freire, Paulo y Antonio Faundez. 2013. Por una pedagogía de la pregunta. Crítica a una educación basada en preguntas inexistentes. Buenos Aires, Argentina: Siglo XXI.

Gadamer, Hans-George. 1993. Verdad y Método I. Salamanca, España: Sígueme.

Kuhn, Thomas S. 2013. La estructura de las revoluciones cientificas. México: FCE.

Latour, Bruno. 2008. Reensamblar lo social. Una introducción a la teoría del actor red.

Buenos Aires, Argentina: Manantial.

Para citar este texto:

Alfaro López, Héctor Guillermo. 2021. "Lectura y conocimiento en Bi-

bliotecología”. Investigación Bibliotecológica: archivonomia, bibliotecología e información 35 (89): 79-90.

http://dx.doi.org/10.22201/iibi.24488321xe.2021.89.58381 